\title{
Platelet-Rich Plasma (PRP) Treatment in Vaginal Penetration Disorders
}

\author{
El Haddad Cynthia ${ }^{1,2,5}$, Darido Jessie ${ }^{3,5}$, , Gharjestani Shamsia ${ }^{4}$, Diari Jed ${ }^{5}$ \\ ${ }^{1}$ Department of Obstetrics and Gynecology, Faculty of Medicine, Holy Spirit University of Kaslik, Jounieh, Lebanon \\ ${ }^{2}$ Department of Obstetrics and Gynecology, The Centre Hospitalier Universitaire Notre Dame de Secours, Byblos, Lebanon \\ ${ }^{3}$ Department of Obstetrics and Gynecology, Faculty of Medical Sciences, The Lebanese University, Beirut, Lebanon \\ ${ }^{4}$ Faculty of Medicine and Health Sciences, University of Antwerp, Antwerp, Belgium \\ ${ }^{5}$ Department of Obstetrics and Gynecology, Centre Hospitalier du Sud Francilien, Corbeil Essonnes, France
}

\section{Email address:}

jesydarido@hotmail.com (D. Jessie)

${ }^{*}$ Corresponding author

\section{To cite this article:}

El Haddad Cynthia, Darido Jessie, Gharjestani Shamsia, Diari Jed. Platelet-Rich Plasma (PRP) Treatment in Vaginal Penetration Disorders. Journal of Gynecology and Obstetrics. Vol. 8, No. 4, 2020, pp. 71-80. doi: 10.11648/j.jgo.20200804.11

Received: May 9, 2020; Accepted: May 25, 2020; Published: June 4, 2020

\begin{abstract}
Vaginal Penetration Disorders (VPD) consist a major sexual disorder that has a significant impression on young ladies and couple's relationships and also consist of a predisposing factor for anxiety disorders. All existing treatments of vaginismus are still a big challenge and often without success. Before performing PRP patients were referred to the gynaecologist, urologist or psychologist. Almost in every case after several attempts to be treated, they were disappointed with the results. Often, they have admitted to accept their problem and keep suffering because no physician could offer any effective help with significant results. We are reporting in this article a serie of ten cases where the Platelet Rich Plasma (PRP) treatment was tried based on their voluntary participation due to particular and relative sexual problems. Based on the results that are exposed here, we can say that PRP clearly has a positive effect not only on rejuvenation of the female genital structurebut also on improving VPD symptoms. It reduces pain, reduces mucosal dryness and chronic fissuration. The duration of the effect on the vaginal structure as well as clinical improvements requires more case studying and research. These patients will be evaluated for the coming 2 years to acquire more data to be analyzed in order to complete our observational study. It is nothing but the beginning of a new era in the sexual dysfunction therapy.
\end{abstract}

Keywords: Vaginal Penetration Disorders, Sexual Disorder, Vaginismus, Platelet-rich Treatment

\section{Introduction}

Vaginismus and dyspareunia currently classified as vaginal penetration disorders (VPD) in the latest edition of Diagnostic and Statistical Manual of Mental Disorders [1]. The aetiology of this disorder is multifactorial, and the pathophysiology is as of yet unknown. This sexual disorder has a significant impression on young ladies and couples which often leads to sexual, psychological and relationship problems. VPD is also a predisposing factor for anxiety disorders and has an emotional influence on women mental health. There is still a taboo atmosphere about this topic, and this prevents women to seek help, nor talk about it.

By definition, Vaginismus is an involuntary contraction of the vaginal muscles which makes sexual intercourse and gynaecological examinations, difficult and sometimes nearly impossible. The significance of vaginismus, has recently been expanded in the light of research and now incorporates pain, muscle tension and fear of penetration. [2]

Vaginismus can be classified as either primary or secondary. Primary vaginismus occurs when the woman has never been able to have penetrative sex because of the involuntary contraction of her vaginal muscles. Secondary vaginismus occurs when a woman has previously been able to have penetrative sex, but is no longer able to. Vaginismus can be also classified as global and partial. In global vaginism nothing can be inserted in her vagina including normal things, and also, she cannot undergo vaginal 
examination. In partial vaginism, the woman can insert a tampon and undergo vaginal examination, but she cannot have sexual vaginal penetration. [3] Some literature proved that vaginal atrophy is a common condition among peri- and post-menopausal women due to experiencing a "pseudo"vaginism. Up to $45 \%$ of postmenopausal women will develop these symptoms. On the other hand, dyspareunia is persistent genital pain during sexual intercourse. The most common cause of chronic superficial dyspareunia is premenopausal vestibulodynia. It is when Vulvar pain is triggered by touch, pressure and vaginal penetration. Dyspareunia not only leads to anxiety disorders but also influences the mental health of women. [4]

There is not much literature to be found about the incidence of vaginism but according to 'Tamara Melnik 2017 there is an estimation of 1 in 500 women who are affected or approximately $1-7 \%$ of females in a worldwide. [2] Another literature source estimates the prevalence of $30 \%$ in primary care and that can be up to $10-20 \%$ of women who seek professional help for a sexual problem, suffer from vaginism. [5]

The aetiology and differential diagnosis of VPD is important for choosing the treatment approach. Based on the correct diagnosis with existing methods, treatments were attempted to help the patients suffering from this problem. Classical treatment approach must be multidisciplinary to ensure better results. Experts in human sexuality consider that the ideal treatment for vaginismus must access the complex interplay between the biological, emotional, psychological and relational components of women's and couples' lives. Therapeutic approaches include psychosocial interventions, drug treatments (including anti-depressants, anxiolytics, local anaesthetics or botulinum toxin A) and combinations of these. The potential benefits and harms of these treatments should be considered, ideally through evidence from high quality randomized controlled trials (RCTs). [2] Systematic desensitization (behavioural therapy aimed at making people less sensitive to certain stimuli), together with the use of dilators (instruments used to systematically dilate body openings, in this case the vagina), is a combination that is often used as therapy for vaginism. The aim is to achieve deep muscle relaxation and the gradual insertion of a dilator or finger into the vagina to reduce the anxiety associated with penetration. Anxiety inhibitors, such as diazepam, in combination with psychotherapy are the most commonly used combination therapy. Medication is often used in women who could not be helped with psychotherapy alone and who are very anxious. Local anaesthesia, such as with a lidocaine gel, was suggested as a possible treatment option, the use of antidepressants or anti-epileptics has already been tried, but without much success. Botulinum toxin (Botox), a temporary muscle-paralyzing agent, could help in the treatment of vaginism with the aim of reducing the excessive contraction of the pelvic floor muscles, but this treatment is still at an experimental stage, and comparative studies are not yet available. There is a general consent that treating vaginism is a challenge. The practical and emotional impact of the exercises and the use of dilators should certainly not be underestimated by healthcare providers, which could otherwise lead to insufficient support and information. Sexologists can play an important role in supporting women and their partners during treatment and can help to improve their communication about sex, sexual satisfaction and increase intimacy between partners. All existing treatments of vaginismus is still a big challenge and often without success. There is not much literature found about treating VPD using PRP specifically, but there are some pelvic and vaginal disorders that have been treated with great benefits. [5]

Platelet-rich plasma (PRP; autologous platelet-rich plasma - APRP) is a body-own-substance that is well tolerated and has a gradual effect which is simple to manage. Besides that, it is also a natural alternative to conventional methods, especially since it is a biological approach. PRP is quick, easy and can be combined with other treatments. [6] PRP are platelet concentrates made of autogenous blood with a high number of platelets in a small volume of plasma. The clinical efficacy of platelet concentrates depends mainly on the number of platelets and the concentration of their growth factors. These will act as transmitters in most tissueprocesses, particularly in healing, where they are responsible for proliferation, differentiation, chemotaxis and tissue morphogenesis. Growth factors derived from centrifuged blood were first used in patients with chronic skin ulcers. [7] The clinical use of PRP for a wide variety of applications has been reported mostly in oral and maxillo-facial surgery, orthopaedic surgery, treatment of soft tissue diseases, treatment of burns, hard-to-heal wounds, tissue engineering and implantology. [8-11] Growth factors are important mediators responsible for wound healing. Plasma has a 5-to 10-times higher concentration of growth factors compared to our blood and tissue, therefore also playing a role in regeneration and rejuvenation. PRP is widely applied in clinical scenarios, even in mucous tissues such as in the eye and the mouth, to improve tissue regeneration. In addition, the combined PRP and mesenchymal stem cells have been widely used lately. [12-16]

The main objective of this study is to find out from the practice point of view, whether PRP can improve the symptoms and signs of women with vaginal penetration disorders/vaginism, to evaluate the symptoms and macroscopic improvement of genital tissue, vaginal mucosa, atrophy, scars etc.

\section{Methodology}

The hereby reported cases presented in a private teaching clinic in Belgium where about 1000 permanent patients and 200 not permanent patients are registered. Note that $90 \%$ of the patients are from Caucasian origin and $10 \%$ have an international background.

The selected women that were included are those who have suffered from vaginal penetration disorders (VPD) or Vaginismus, and who have never been able to receive 
successful treatment from their own doctors or specialists.

An advertisement was put in the waiting room of thisclinic with information about VPD and the regenerative method of treatment regarding this case study.

Semi structured qualitative interview were done amongst 10 women between the ages of $39-71$ years old. A selection was done without minding their menopause, pre menopause and physical sexual trauma except for one patient who was physically traumatized at her vaginal structures. There was also no consideration of their method of child birth.

Women were asked about excessive use of vaginal lubricants, vaginal cleaning products, soaps, local anaesthesia or any other drugs. Everyone was asked about penetration problems and use of other sexual alternatives like oral and anal sex for better evaluation of psychological problems. They were asked if they experienced an orgasm, state of their libido and bisexual or heterosexuality. None of them were bisexual.

Almost all women had the similar symptoms during coitus, pain during penetration, burning sensation, spasms, vaginal dryness, and urinary complaints.

The lifting rejuvenation of the vaginal canal and labia's will also be evaluated. Using digital vaginal examinations the spasm, lubrication and softness of vaginal tissue will also be evaluated and compared with the original state. Next to that, the study will compare the psychological effect of the participants and their sexual partners during the time coming.

Questionnaires (figure 3) were filled in by patients and their partners together. Afterwards we went over their answers to be sure that the patients understood the questions well and answered correctly.

The choice of high quality PRP with maximum amount of Plate cell was made from Arthrex based on literature. We did not mix any Natrium Citrate, based on our experience natrium citrate gives a burning sensation.

Before PRP treatments, patients were informedabout the procedure. Each of them had to sign an informed consent form, showing that she agreed to take part in the treatments.

The steps were as follow:

a) Installation of the patient on the examination table.

b) Clinical examination of the patients' genital / vaginal area.

c) Emla cream was locally used on the vaginal wall to ensure local anaesthesia for those who required it.

d) Documenting of lesions via pictures and videos.

e) Preparing PRP.

f) Performed PRP.

g) 5.0-6.5 $\mathrm{lm}$ PCT PRP collected.

h) Injections are done submucosal, deep submucosal, intramuscular in the area of visible and palpable (touchable) spasm, hardness of tissue and preclitoralclitoral region.

Participants were given "homework" to start exercising regular vaginal sex with possible penetration as a way of dilating the compromised mucosa.

The psychological state was prepared of these women by counselling them. This is to help them conquer their trauma of anxiety and pain, which they had before PRP, during coitus and let them experience normal sexual life.

Table 1. Questionnaire filled in by patients and partners.

\begin{tabular}{|c|c|c|c|c|}
\hline Age & Symptoms since onset of sex life & $\begin{array}{l}\text { Before PRP } \\
\text { Treatment }\end{array}$ & $\begin{array}{l}4 \text { weeks after 1st } \\
\text { treatment }\end{array}$ & $\begin{array}{l}8 \text { weeks after 1st } \\
\text { treatment }\end{array}$ \\
\hline & $\begin{array}{l}\text { Have you had psychological sex trauma in the past? } \\
\text { How long have you been bothered? } \\
\text { Your current partner is the 1st? } \\
\text { Problem with sex with current partner (2nd or 3rd)? } \\
\text { Problem during intercourse with 1st partner? } \\
\text { Symptoms of onset sexual life? } \\
\text { Symptoms later sexual life? } \\
\text { Congenital vaginal deformity? } \\
\text { Libido problems? Desire sexual contact? } \\
\text { Had vaginal childbirth? } \\
\text { Have you had vaginal surgeries? } \\
\text { Do you use local hormonal medicine (in the vagina)? } \\
\text { Burning sensation during intercourse? } \\
\text { Bleeding during intercourse? } \\
\text { Dry vaginal canal during intercourse? } \\
\text { Do you Have vaginal symptoms affecting your sexual life? } \\
\text { Do you have constriction feeling vaginal during intercourse? } \\
\text { Does your partner have problems penetrating? } \\
\text { Do you have anal penetration (sex)? } \\
\text { Do you have problems with anal penetration? } \\
\text { Do you have vaginal orgasm? } \\
\text { Have you ever had anal orgasm? } \\
\text { How often do you have vaginal intercourse per month? } \\
\text { Do you use vaginal narcotic ointment for intercourse? } \\
\text { Are you using vaginal lubricant during intercourse? } \\
\text { Do you masturbate? } \\
\text { Do you Use a lot of disinfection, soap other products for vaginal cleaning? } \\
\text { Are you bisexual (Masculine and feminine partner)? }\end{array}$ & & & \\
\hline
\end{tabular}


Evaluation pictures of tissues are made 4-6 weeks after the first treatment as well as videos of interview of improvement of their clinical symptoms as well as about their satisfaction

Next stage is performing of the second treatment ${ }^{1}$ only in cases where there is not a $100 \%$ clinical improvement, which was done 5-8 weeks after first PRP treatment.

\section{Results}

In only 2 weeks time after placing the invitation of participation to the case study in the waiting room of the clinic, we received 7 responses from potential candidates, and we still keep on receiving requests from new candidates to participate.

During the interviews with these women and some of their partners, we found out that a lot of women are ashamed, fedup or have given up to speak about these problems, and often the initiative came from their husbands to talk to me about their vaginism.

Some of the women said spontaneously "He is allowed to have another sex partner, "I understand even if it can hurt me "I understand my husband's physical need, because I cannot offer him sex with penetration."Another patient said:" $\mathrm{He}$ does not believe I suffer; he thinks I play a game and that I don't want to have sexual intercourse with him."Some women believed that "they were some kind of disabled person, not a real woman', and believed 'they had some natural defects'. Some husbands understood their wife, and they adapted to the situation. They used other methods to get an orgasm, but for a lot of men penetration remains important.

We heard a lot of frustrations during the interviews from the women as well as from their partners

Note that the final data analysis will be provided at the end of the study in 2 years time. For now there are only the preliminary study results, since this is still an ongoing study.

We found out that half of the women sexual partners were interested to talk about this problem after seeing the advertisement in the consultation waiting room. Most women were ashamed. They had some kind of complex and had the feeling that there was no solution available for their problem. They felt that there was no need in participating because it would not work for them. Basically they had given up and accepted their condition as being permanent and untreatable.

Some of the women had guilty feeling towards their partners thinking that they were the cause of scarcity of sex in the life of their partner.

Other women had psychologically accepted the idea to allow their men to look for other sexual partners.

For others they used other sexual alternatives, mainly oral and anal sex to satisfy their partners even though it was not pleasant for them.

1So far 3/10 patient received the second treatment yet, the rest will follow in the coming time until determined study duration, which is 2 years $(06 / 2021)$.
During the evaluation after the first PRP treatment, almost all women were frustrated with the fact that, they have suffered for a long time and nobody had ever proposed this kind of treatment before. We heard from many women that their husbands were very thankful. PRP has not only given them vaginal and sexual comfort, but also their functional urinary symptoms were improved. For other women their sport activities like bicycling were resolved and had no more vaginal pain or bleeding.

One out of 10 women, beyond the improvements of VPD symptoms, said that her vaginal canal was tighter. Moreover she was the youngest in the group. She wanted her vaginal canal to be wide and soft.

At this moment, many women in the study have no pain anymore, but they must defeat their anxiety and fear of initiating sex.

\subsection{Case \#1 (Figure 1, 2)}

A 64 year old woman, mother of 2 children with normal vaginal delivery presents with the following:

"I always had a normal sexual life until 30 years ago when slowly I started feeling pain during coitus. I felt spasm during penetration, dryness and pain. I started being afraid and avoided sex. For 30 years I did not have vaginal penetration anymore. In the past 5 years I have undergone an operation for uterine prolapse and bladder prolapse. After the operation I had chronic urinary problems, infections, pains and frequent micturition". There is atrophy, chronic fissures, scars, muscle spasm and dry mucosa.

8 weeks after the second PRP-session:

"I received 2 treatments with an interval of 4 weeks. The 4th week after the second PRP my local vaginal symptoms during coitus were reduced to $78 \%$ and I now have regular penetration once a week, there is no severe dryness anymore. I now experience sexual pleasure and even my chronic urine symptoms have been reduced. I still don't understand how this is possible. I feel so ashamed and frustrated that I suffered for so many years and visited so many specialists while the problem is so easily solved".

\subsection{Case \#2 (Figures 3, 4)}

A 71-year old woman with 4 normal vaginal deliveries, one of which had ruptured the vaginal vestibulum and labia minora, 35 years ago. She developed virginal scars and slowly vaginal spasm and pain.

"I have a normal libido, but I try to avoid vaginal intercourse because of the pain, bleeding and severe dryness. I sport a lot and after long biking I always had vaginal bleeding. I appreciate my husband because he always understood and supported me."

4 Weeks after one treatment:

"I feel very comfortable, no pain and bleeding after mountain biking. I do not have sex yet because my husband is not sexually active, but please look no fissure seen for a young one for me." 


\subsection{Case \#3 (Figures 7, 8)}

43 years old woman with caesareans section.

"From the first day of my sexual life I had pain, spasm and dryness during penetration.

I am married for 9 years and I use EMLA cream and lubricant to help me. I have a normal libido, but I am afraid of the pain, me and my husband try to have minimum vaginal penetration. I feel guilty towards my husband because I cannot satisfy him sexually. I have seen a specialist many times and nobody could help me. Two years ago, my gynaecologist removed the remaining part of my hymen with the purpose of widening my vaginal canal, but this didn't have an effect in any way.

In September 2018 fortunately I talked about this to my general Physician, and I am so happy that I received my first PRP treatment and after 5 months the second treatment.

Four weeks after the second PRP-session, I can say that $95 \%$ of my complains have gone. I enjoy sexual penetration, but I have to work on my fear before initiating sex. I am so happy and thankful that I shared this problem with my GP".

\subsection{Case \#4 (Figures 9, 10)}

A 59 year old woman, who had normal vaginal delivery of 2 children.

"From the beginning of my relationship I had no comfort during sex. My present partner is also my first partner. In the past 15 year after the beginning of my menopause I started to experience vaginal discomfort, pain, dryness and spasm and narrowing of the vaginal canal. I have never had an orgasm. We tried once every 5 years to have vaginal sex, but we have never succeeded. I love my husband and I have a normal libido. I gave my husband a permission to have sexual relationship with another woman because I feel guilty."

After PRP treatment,

Clinical examination: significant improvement of the mucus colour, feels softer and les irritable during examination, lifting of labia major. She feels much more comfortable, but she has big fear of sexual contact and so far, she has not tried.

We did plan a psychological assistance approach for her and will be coaching both partners over the coming two years.

\subsection{Case \#5 (Figures 11, 12)}

A woman of 50 years of age with $1 \mathrm{C}$ section who had primary Vaginism.

"Since the beginning of my sexual life, I experience burning and a painful sensation during vaginal coitus. My husband adjusted himself to my complaints, so we had sex only when we were on holiday. While laughing she asked her husband if he had another woman?"

Result: 6 weeks after treatments:

"My husband is so thankful that you proposed this treatment. We lost 30 years of my young age where I could enjoy sex". Why did no medical helper advise me to getthis treatment?

\subsection{Case \#6 (Figures 13, 14)}

A 60 years old woman with multiple vaginal deliveries and sexual physical trauma in the past (the only woman in the group).

"The last orgasm, that I ever had, was when I was 20 years of age. I lived in one house with 2 partners in agreement between us 3. 19 year ago, I had breast cancer which affected my sexual health. One of my partners passed away 9 years ago since that time; I had in last 7 years only 3 times vaginal penetration which was very painful, burning sensation and even bleeding from my old scars. I feel guilty for my partner and I would like to feel better and let him enjoy."

3 weeks after first PRP treatment: you can see tenderness in the scars, area is softer and changed colorization of the mucosa. "I did not have sex yet, because of my low libido and psychological barriers". We advised her to start having vaginal penetrations in order for her to see is she would feel pain and strangling.

6 weeks after first PRP: "I feel very comfortable in my genital area, even my urine problems are better. No more feeling of not being able to empty my bladder or a false need to urinate. Me and my husband have had vaginal intercourse after so many years, all gone softly, no dryness, no pain at all and no burning sensation afterwards. But I have not had an orgasm yet." This she said with a smile on her face.

\subsection{Case \#7 (Figures 15, 16)}

A 39 year old woman with two vaginal deliveries and primary vaginism.

"I had penetration problems since the first day of my sexual life. My husband is my first partner. I don't have a high libido. I never had experienced an orgasm in my life. I have pain, dryness, burning sensation and spasm of the vaginal canal so that my husband has difficulties to penetrate. I have intercourse for about 3 times a month on my husband's request. I wish that I also could get better and enjoy sex."

After 4 weeks: macroscopical improvement of mucosa colour, more softness of tissue. Her labia minora are reduced in size. She feels more elasticity; Pain sensations are $40 \%$ reduced. Vaginal contact less irritable for her. She has no changing in her libido yet. She would love to repeat PRP even if she is very afraid of infections.

\subsection{Case \#8 (Figures 17, 18)}

woman of 56 years old with no children.

"I have had VPD in the last 10 years, which I didn't have before with my first partner. I have dryness, pain and burning sensation during vaginal penetration without spasm. I use a lot of vaginal cleaning products and lubricants that my GP advised me to stop.

Results: 4 weeks after: big changing in softness, less dry, no sex yet because she is afraid. Macroscopic you can see more healthy colour of mucosa, no visible fissuration. 


\subsection{Case \#9 (Figures 19, 20)}

A woman of 55 years old, mother of 2 kids delivered with caesareans section with multiple sex partners in the past.

"Since one and half years ago, I experienced severe dryness fissures, burning sensation and narrowing of vaginal canal, not only by vaginal penetration but also with the use of tampon. My husband can now penetrate without any impediment. I use lubricants and have sometimes an orgasm. I appreciate my present partner and would like to have normal sexual life together".

Four weeks after the first PRP: "I feel comfortable in my vaginal region; there is significant decrease of dryness and burning sensation. We have more sexual penetration than before.

My symptoms have improved for about $30-40 \%$. The fear had an important place in my sexual life, since it prevents me from spontaneously having sexual penetration. I do feel more tightness around my vagina. 4 weeks after my first PRP I received my second session: $70 \%$ symptoms are better, vaginal outlook is rejuvenated. Labia minora are smaller in size.

\subsection{Case \#10 (Figures 21, 22)}

71 Years old, for 3 years VPD, dryness, pain. Both partners wanted the treatment because vaginal penetration was not possible all the time. They wanted to enjoy their sexual life further;

Results: 3 weeks after treatment: "I do feel $80 \%$ improvement, and feel very comfortable, not as dry as it was before. More comfort during vaginal coitus."

Visible fissuration and irritation colour on vaginal entry are gone, significant decrease of dryness and burning sensation.

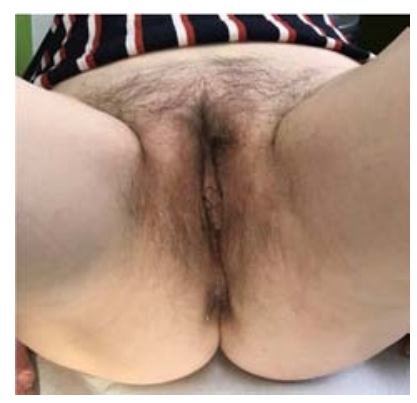

Figure 1. Patient with atrophy, chronic fissures, scars, muscle spasm and dry тисова.

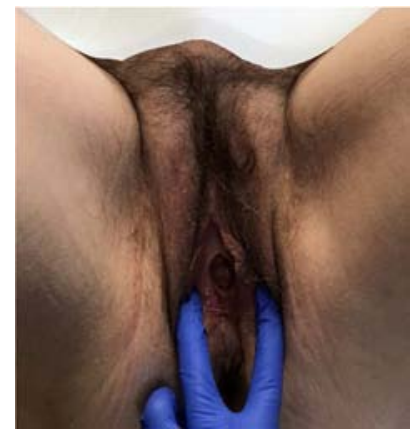

Figure 2. Five weeks after first PRPimprovement mucosal condition and rejuvenation of labia major.

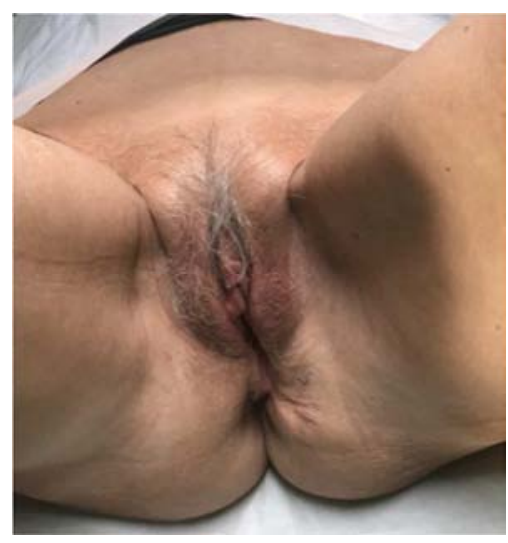

Figure 3. A symptomatology of pain, bleeding and severe dryness.

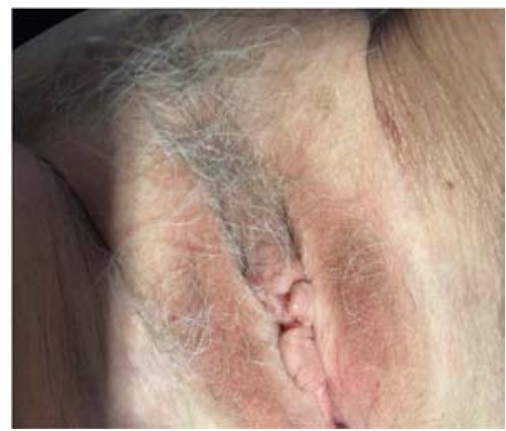

Figure 4. After 4 weeks; see the quality of mucous membrane (colour atrophy and dryness).

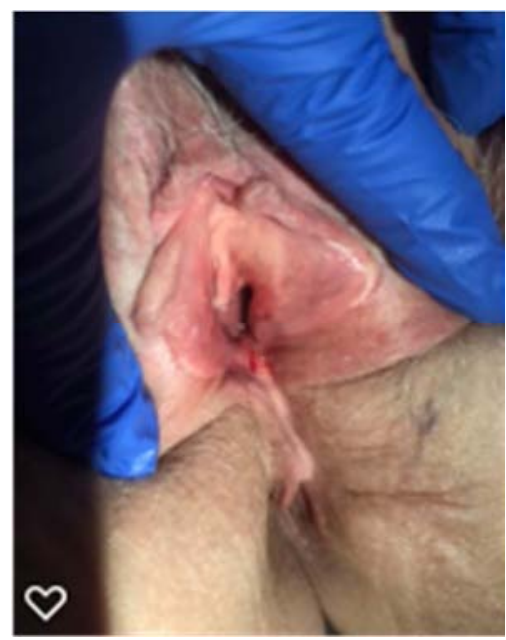

Figure 5. Before the PRP, presence of fissure.

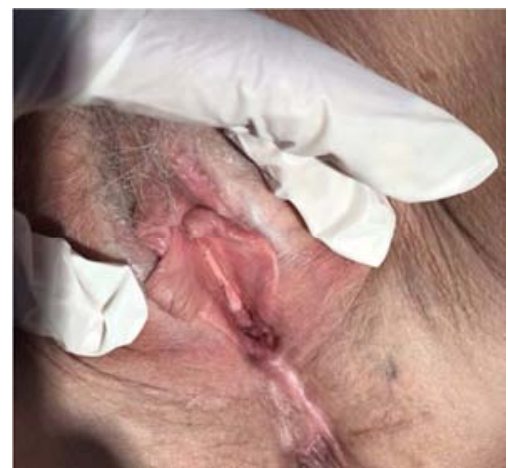

Figure 6. After PRP, No fissure seen. 


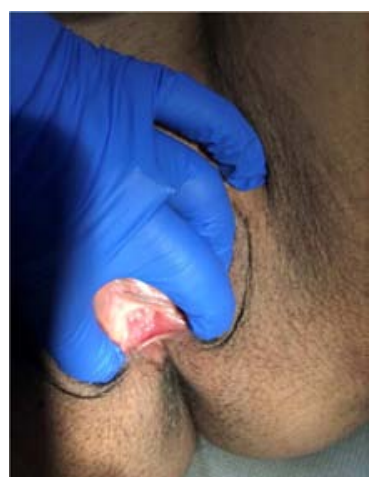

Figure 7. Before PRP treatment: pain, spasm and dryness during penetration.

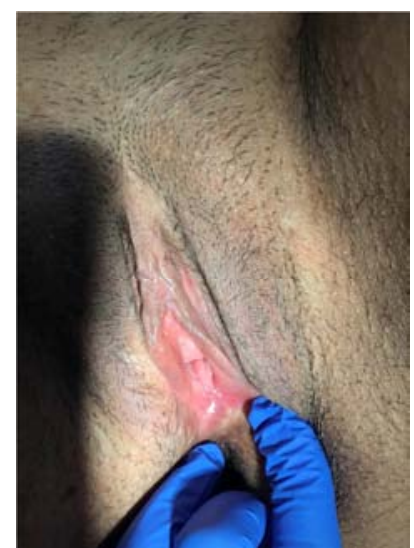

Figure 8. Four weeks after the second PRP-session.

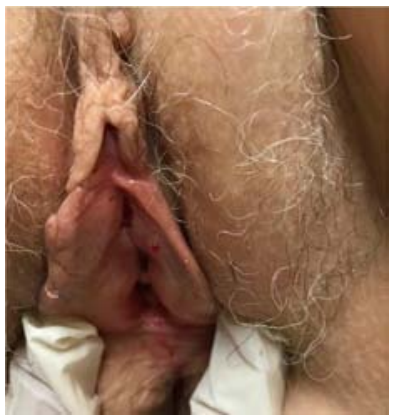

Figure 9. Before PRP treatment: narrow vaginal canal.

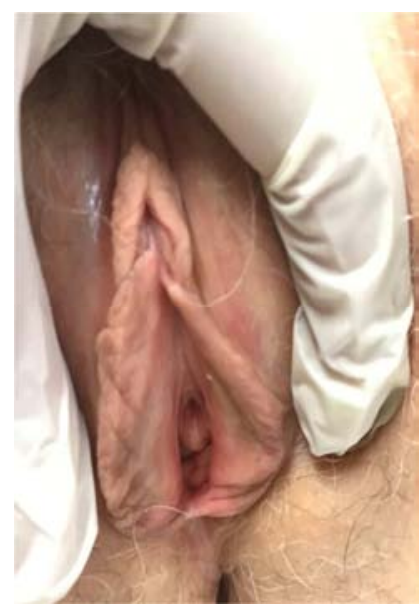

Figure 10. After PRP treatment: Significant improvement.

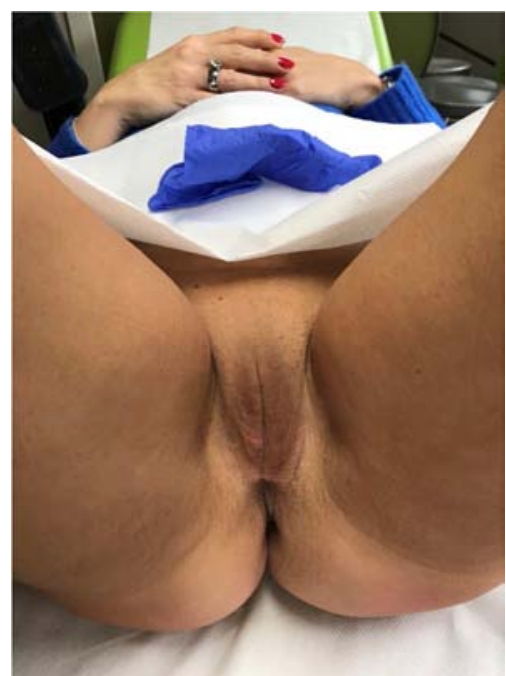

Figure 11. Before rejuvenation of labia major.

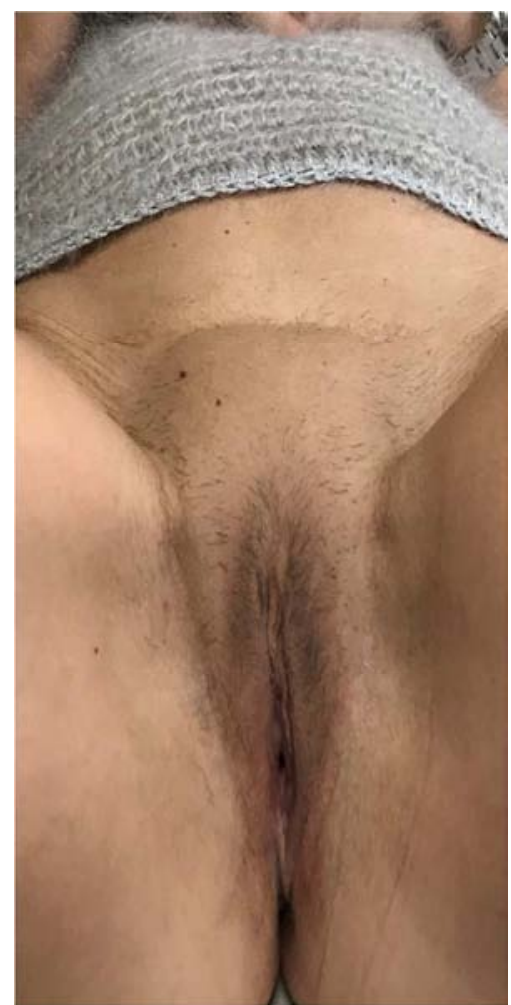

Figure 12. Sixweeks after treatments, lifting effect to seen on labia.

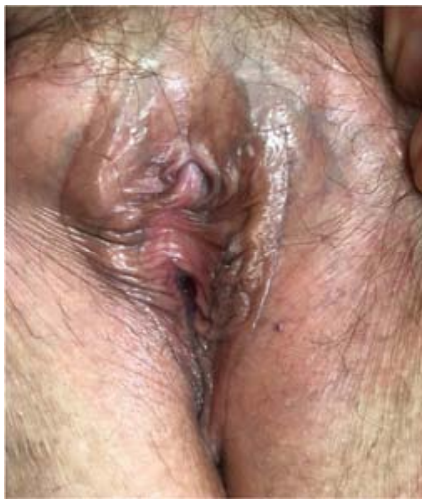

Figure 13. Before PRP treatment. 


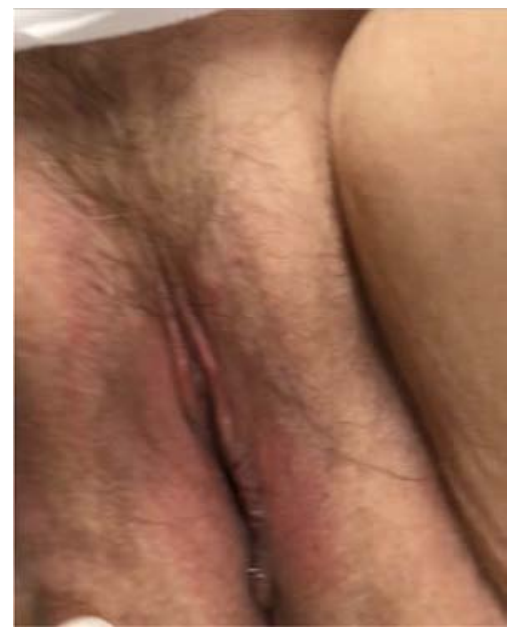

Figure 14. Lifting of tissue, better and healthier colour of mucosa, less oedema and even varicosities, less redness.

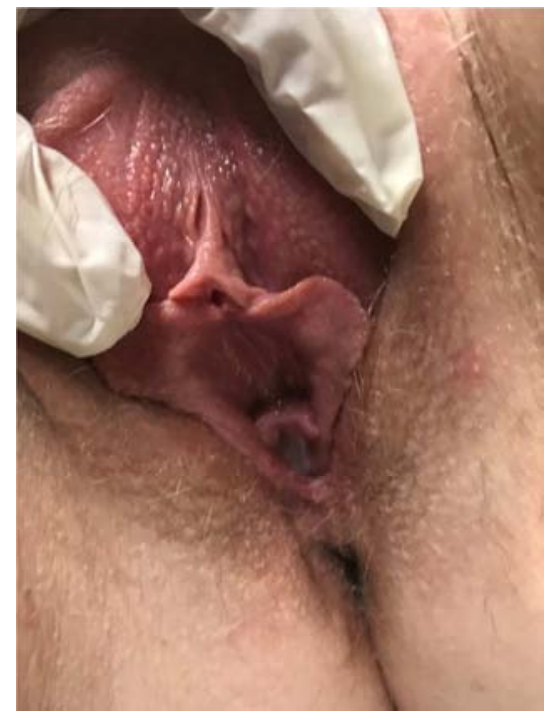

Figure 15. Before PRP treatment.

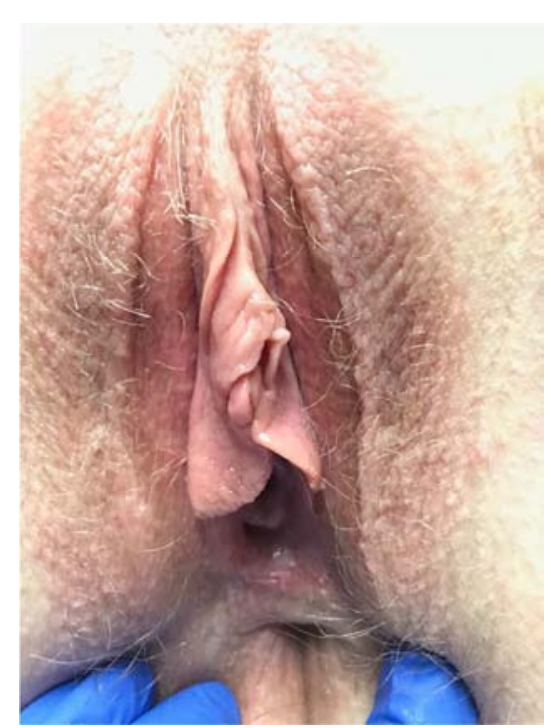

Figure 16. Can notice by clinical examination improving colour of mucosa, no sign of new fissuration, Labia looking smaller and lifted.

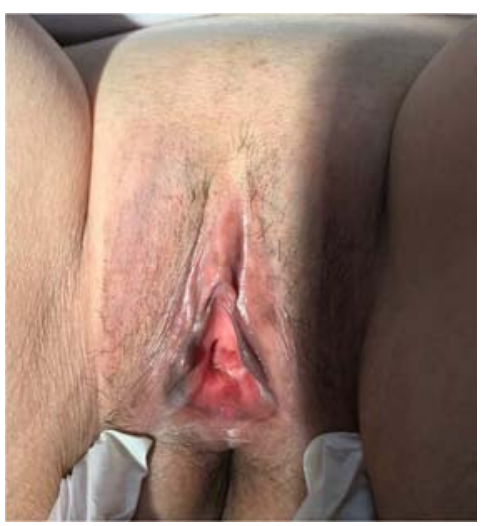

Figure 17. Before PRP treatment.

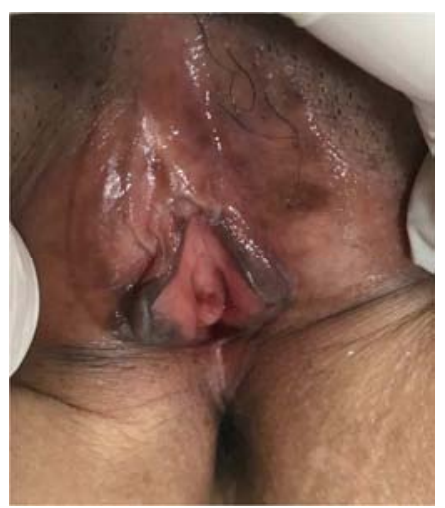

Figure 18. More healthy colour of mucosa, no visible fissuration.

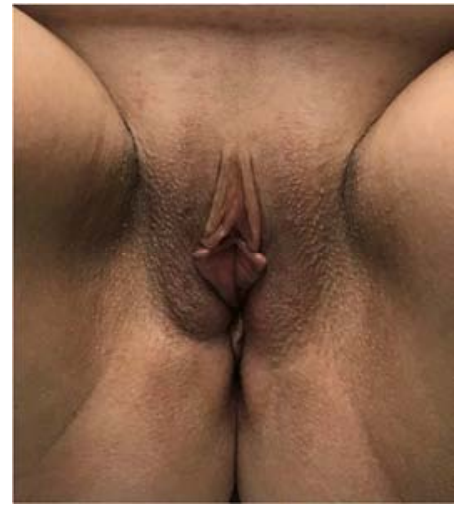

Figure 19. Before PRP treatment.

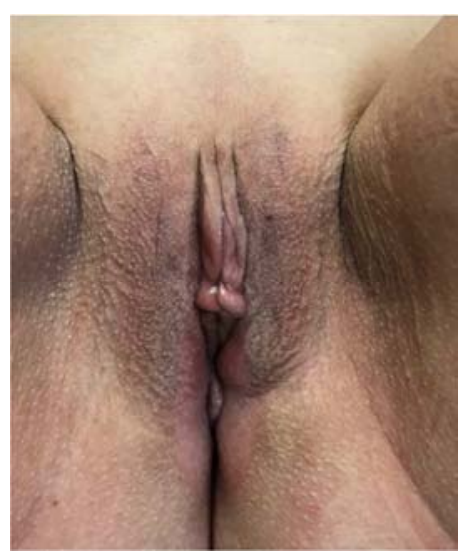

Figure 20. Vaginal outlook is rejuvenated. Labia minora are smaller in size. 


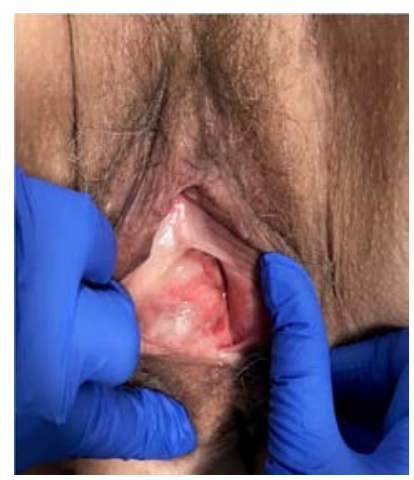

Figure 21. Before PRP treatment.

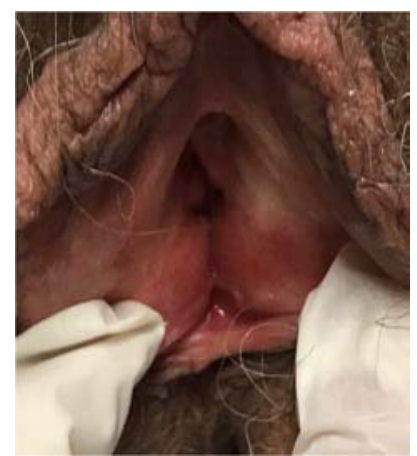

Figure 22. Visible fissuration and irritation colour on vaginal entry are gone.

\section{Discussion}

This might mean there are far more women with mild to severe VPD problems than we expected. These women have accepted their symptoms and they have stopped seeking medical help. Sometimes they have psychological -social barriers which prevent them for asking help. I noticed that the high price of the treatment is also a threshold for seeking help. This treatment is not covered by Belgium medical insurance companies.

Some of them suffer from serious psycho-emotional problems based on VPD.

An important part of physical problems don't only occur during sexual intercourse but also during sport activities (cycling, spinning), and urogenital discomfort during their daily life.

These patients are in a vicious circle of sex-pain and burning sensations and the fear not being able to satisfy their sexual partner, which has a negative impact on their relationship.

Another challenge after the PRP treatment is to get these women released from their fear to have a normal sexual life. So far, no psychologist was approached, but husbands should be explained and shown evidence pictures of their partner's lesions. They will play a major role by understanding andcollaboration to revalidate and help their wives escape from the vicious circle sex $=$ suffering $=$ fear.

After the performance of the treatment session of PRP and evaluation of each case, the following questions arose and are related directly to VPD problem:
1. What about the psychological improvement and satisfaction in the relationship, sex life?

2. How many sessions of PRP are needed to have maximum efficient effect and resolving symptoms which are causing the avoidance of vaginal coitus?

3. How much time intervals are needed, are better between each session?

4. How long will they remain symptom free during the 2 years of observation? Is there any chance of full healing/recovery?

Until the moment this article was written, there is no medical literatureavailable on the effect of PRP in VPD and its lasting clinical effect, as well as the macroscopic results in each case. Therefore, these patients should be followed up during the upcoming 2 years.

As for the case where beyond the improvements of VPD symptoms, the women felt that her vaginal canal was tighter, this can be explained by the fact that plasma can improve the quality of vaginal tissue by filling up the empty extracellular matrix. Moreover, due to the fact that she was the youngest in the group, the result can consequently be related to the vaginal structure where there is still no atrophy of the muscles. This made us think of changing the PRP injection technique by not injecting in a circularly motion to the whole vagina but to skip tissues and evaluate during follow up. This technique is to see if the vagina canal would become smaller or not.

The further evolution of this study is to follow these women up in different aspects; subjective vaginal complains quality of sexual life, evaluation of their psychological fear for vaginal penetration.

Furthermore, the quality of anatomy, structure, scars, atrophy, secretion, muscle spasms and tonus with the help of taking pictures before and after must be evaluated. The vaginal clinical and digital examination will be done and finally a comparison will be made.

Since this work is done in the context of a GP practice, which is the first line health care, there were no extra technical medical investigations like taking biopsy, vaginal pressure measurements, pelvic floor muscle control etc.

\section{Conclusions}

Sexual disorders have a significant impression on young ladies and couple's relationships and also consist of a predisposing factor for anxiety disorders. All existing treatments of vaginismus are still a big challenge and often without success.

Before performing PRP patients were referred to the gynaecologist, urologist or psychologist. Almost in every case after several attempts to be treated, in the second line medicine, they were disappointed with the results. Often, they have admitted to accept their problem and keep suffering because no physician could offer any effective help with significant results.

The positive effect that was achieved was not only macroscopic but also an improvement of the symptoms. Gaining very effective results almost in all cases made us 
report them aiming to get practical evidence of the efficiency of PRP. PRP can help patients with a product from their own body, with best results, without any danger or side effects. PRP is not an invasive treatment without risk or side effects and can be perfectly tolerated by the patient. PRP clearly has a positive effect on rejuvenation of the female genital structure.

PRP has a significant effect on improving VPD symptoms. It reduces pain, reduces mucosal dryness and chronic fissuration. By reducing the complaints of VPD there is a huge improvement in the sexual relationship between partners.

A good psycho-emotional support and a good mental preparation for the patient are important to realize that sexual intercourse is possible without suffering and fear. Support and information of partners during treatment can help them communicate better about sex, increase intimacy and improve the sexual satisfaction of their relationship.

The amount of PRP treatments needed for a maximum result for the vaginal structure has yet to be determined and requires more case studying and research. 8/10 patients already showed major improvements after just one treatment and were able to have sexual intercourse, which improved their sexual relationship with their partner. The duration of the effect on the vaginal structure as well as clinical improvements requires more case studying and research.

These patients will be evaluated for the coming 2 years to acquire more data to be analyzed.

\section{References}

[1] The diagnostic and Statistical Manual of Mental Disorders, $5^{\text {th }}$ ed.; DSM-5; American Psychiatric Association, 2013

[2] Melnik T, Oswaldo M. Vaginismus is ruining sex. Research must move beyond penetration. Jnr for evidently Cochrane, 2017.

[3] Van der Velde J, A Psychophysiological Investigation of the Pelvic Floor. The Mechanism of Vaginism, Thesis university Amsterdam, 1999.

[4] Konstantinos H, Hatzichristou D Sexual Dysfunctions: Classifications and Definitions; J Sex Med, 2007 Jan; 4 (1): 241-250.
[5] Ulrich S., Schnyder-Lüthi C., Ballinari P., Blaser A. Therapy for Vaginismus: In Vivo Versus In Vitro Desensitization. Can J Psychiatry. 1998 Nov; 43 (9): 941-4.

[6] Elghblawi E. Platelet-rich plasma, the ultimate secret for youthful skin elixir and hair growth triggering. J Cosmet Dermatol. 2018 Jun; 17 (3): 423-430.

[7] Seok H., Eun S., Tae H. Rejuvenation Using Platelet-rich Plasma and Lipofilling for Vaginal Atrophy and Lichen Sclerosis.Journal of Menopausal Medicine 2017; 23: 63-68

[8] Yuvika R., Sujata M., Mahesh V. et al. Platelet-rich fibrin: the benefits. British Journal of Oral and Maxillofacial Surgery 54 (2016) 57-61

[9] Shu-Hung H., Sheng-Hua W., Su-Shin L., et al. Platelet-Rich Plasma Injection in Burn Scar Areas Int J Med Sci. 2018; 15 (3): 238-247.

[10] Alleviates Neuropathic Scar Pain. International Journal of Medical Sciences2018; 15 (3): 238-247.

[11] Xiaolin H., Yan Ze., Rongrong Z., et al. Using platelet-rich plasma for the treatment of symptomatic cervical ectopy. International Journal of Gynecology and Obstetrics 119 (2012) 26-29.

[12] Osaid H. A., Ioannis G. The evidence behind the use of platelet-rich plasma (PRP) in scar management: Scars, Burns \& Healing Volume 4: 1-15. 2018.

[13] Yajie C., Jingjie L., Yuqing C., et. Al. Autologous platelet-rich plasma promotes endometrial growth and improves pregnancy outcome during in vitro fertilization. International Journal of Molecular Sciences. 2015; 8 (1): 1286-1290.

[14] Franka K., Tanja S., Melanie U., et al. Comparative Analysis of Different Platelet Lysates and Platelet Rich Preparations to Stimulate Tendon Cell Biology. Int J Mol Sci. 2018 Jan 10; 19 (1). pii: E212.

[15] John W. Platelet-rich therapies for musculoskeletal soft tissue injuries. Cochrane Database Syst Rev. 2013 Dec 23; (12): CD010071.

[16] Tasmania del P., María M. T., Isabel A., et al. Platelet-rich plasma for the treatment of diabetic foot ulcers. Wound Repair Regen 2019 Mar; 27 (2): 170-182. 